\title{
Large Eddy Simulation of Flow and Tracer Transport in Multichamber Ozone Contactors
}

\author{
Dongjin Kim${ }^{1}$; Doo-II Kim²; Jae-Hong $\mathrm{Kim}^{3}$; and Thorsten Stoesser ${ }^{4}$
}

\begin{abstract}
Three-dimensional numerical analyses of flow and transport characteristics in two representative multichamber ozone contactor models with different chamber width were conducted using large eddy simulation (LES). Both time-averaged and instantaneous flow patterns suggest that the flow is characterized by the occurrence of large turbulent structures leading to extensive short-circuiting between chambers and internal recirculation inside the chambers. The flow is also found to be highly three-dimensional, as secondary vortices and recirculation zones develop. The simulation results further suggest that the hydrodynamics in ozone contactors can be improved by reducing the chamber width. The results of the LES are qualitatively verified using previously reported tracer test results obtained from laboratory experiments. The LES technique, applied to the ozone contactor flow and transport of a tracer for the first time, is expected to serve as a powerful tool for existing reactor flow diagnosis, reactor retrofitting as well as for new reactor design.
\end{abstract}

DOI: $10.1061 /(\mathrm{ASCE}) \mathrm{EE} .1943-7870.0000118$

CE Database subject headings: Ozone; Hydrodynamics; Recirculation; Computational fluid dynamics technique; Simulation; Probe instruments.

Author keywords: Ozone contractor; Hydrodynamics; Short-circuiting recirculation; Computational fluid dynamics (CFD); Large eddy simulation (LES); Residence time distribution (RTD).

\section{Introduction}

Past studies have suggested that the hydrodynamics in an ozone contactor has significant impact on its process efficiency (Kim et al. 2004; Tang et al. 2005; Kim et al. 2007). A plug flow is the preferred flow condition due to the absence of flow recirculation, with the consequence of no fluid shear stresses and hence no turbulence. The residence time of all pathogens in a plug flow can be determined exactly from the length-of-the-flowpath and the average velocity. If the residence time is known, the ozone dose can be specified accordingly hence maximizing the inactivation of pathogens such as Cryptosporidium parvum oocyst and therewith minimizing the formation of undesired disinfection by-products such as bromate. Consequently, a meandering plug flow through multiple chambers in series, typically divided by vertical baffles, has been the target flow condition for most ozone disinfection reactors. Despite the intent of the design, however, nonideal flow conditions exist in most multichamber ozone contactors, i.e. a

${ }^{1} \mathrm{Ph} . \mathrm{D}$. Candidate, School of Civil and Environmental Engineering, Georgia Institute of Technology, 790 Atlantic Dr., Atlanta, GA 30332.

${ }^{2}$ POSCO E\&C, Daerung Building 826-20, Yeoksam-Dong, GangnamGu, Seoul 135-769, South Korea.

${ }^{3}$ Associate Professor, School of Civil and Environmental Engineering, Georgia Institute of Technology, 200 Bobby Dodd Way, Atlanta, GA 30332 .

${ }^{4}$ Assistant Professor, School of Civil and Environmental Engineering, Georgia Institute of Technology, 790 Atlantic Dr., Atlanta, GA 30332 (corresponding author). E-mail: thorsten@ce.gatech.edu

Note. This manuscript was submitted on December 30, 2008; approved on June 10, 2009; published online on June 17, 2009. Discussion period open until June 1, 2010; separate discussions must be submitted for individual papers. This paper is part of the Journal of Environmental Engineering, Vol. 136, No. 1, January 1, 2010. CASCE, ISSN 07339372/2010/1-22-31/\$25.00. significant deviation of the hydrodynamics from that of plug flow, due to the presence of turbulence.

A recent three-dimensional laser induced fluorescence (3DLIF) study by Kim et al. ("Investigating multi-chamber ozone contactor using 3D laser induced fluorescence," J. Am. Water Works Assoc., in review, 2009) suggested that flow irregularities such as short-circuiting (i.e. a faster flow through a part of the reactor), internal recirculation within the chamber, and dead zone formation (i.e. area of the reactor with insufficient mixing) could be prevalent in multichamber ozone contactors. In particular, the channel width (i.e. the distance between consecutive baffle walls) was identified as one of the critical design factors that determined the extent of short-circuiting and recirculation. A corresponding reactive transport model simulation further suggested that these flow conditions would have a significantly negative impact on the overall disinfection efficiency of the multichamber reactor (Kim et al., "Investigating multi-chamber ozone contactor using 3D laser induced fluorescence," J. Am. Water Works Assoc., in review, 2009). Heathcote (1995) conducted tracer studies for several hanging baffle designs based on a down scaled ozone contactor model and found that an efficient baffle arrangement would have a significant enhancement in tracer residence time. A Laser Doppler Anemometer study by Shiono and Teixeira (2000) also suggested that a multichamber reactor used for chlorine disinfection with a horizontally meandering flow could also be susceptible to the formation of recirculation zones within the chamber. The above evidences were obtained from a limited set of experiments performed using lab-scale model reactors, thus the extrapolation of the findings to a wider range of design options and operating conditions is inherently limited.

Computational fluid dynamics (CFD) models have been applied to verify the existence of these flow irregularities in multichamber reactors, with the ultimate goal of developing a versatile tool for reactor flow diagnosis and design. Two-dimensional CFD 
simulations with a number of different turbulence models (e.g., depth-averaged viscosity model, $k-\varepsilon$ model, and Smagorinsky model) and different convection schemes (e.g., first- or thirdorder upwind schemes and QUICK scheme) have been attempted for flow simulation of multichamber chlorine contactors (Wang and Falconer 1998a,1998b; Gualtieri 2006a,b, 2007). For multichamber ozone contactors, Henry and Freeman (1995) applied a two-dimensional finite element analysis based on the Reynoldsaveraged Navier-Stokes (RANS) equations and standard $k-\varepsilon$ turbulence model. They found that an additional guide vane would improve the disinfection performance more than corner and edge curvature modifications. A three-dimensional CFD simulation was conducted by Murrer et al. (1995) to investigate mixing behavior at three different spanwise locations by modifying the position of gas injection in an existing ozone contact tank.

Past CFD simulations on ozone contactors have been exclusively based on the RANS equations, in which only the timeaveraged velocity field is computed and all the unsteady effects of turbulence are accounted for by turbulence models. RANS modeling is appropriate in flows where the instantaneous flow is similar as the time-averaged flow. However, the complexity of turbulence in flows that are dominated by large-scale turbulent structures poses a significant challenge to any RANS turbulence model. For instance, the flow around bluff bodies (e.g., baffle walls in a multichamber reactor) may not be accurately simulated by a RANS equations based model (Rodi et al. 1997).

An alternative to RANS-based CFD models is the method of large eddy simulation (LES). LES lies between two extremes of CFD approaches, i.e. models based on the aforementioned RANS equations and direct numerical simulation (DNS). DNS calculates all turbulent scales (spatial and temporal) directly; therefore DNS involves extremely high computational costs, which makes this an impractical choice except for simple geometries or fundamental studies of turbulence. In contrast, LES aims at directly simulating large-scale flow structures that are affected by the geometry and boundaries, and models only the small scales. Hence, LES offers a substantial increase in accuracy over time-averaged approaches, particularly when momentum transfer processes are dominated by large-scale turbulent structures (Rogallo and Moin 1984) at much less computational cost than DNS. Furthermore, given the required relatively high spatial and temporal resolution, LES delivers an enormous amount of information on the mean and instantaneous flow field.

The objective of the present study is to employ the LES method for the first time to simulate the unsteady and complex turbulent flow behavior in open channel, multichamber ozone contactors. LES is of particular interest, since the experimental work by Kim et al. ("Investigating multi-chamber ozone contactor using 3D laser induced fluorescence," J. Am. Water Works Assoc., in review, 2009) suggested that the flow in multichamber reactors was governed by large-scale turbulent structures leading to internal recirculation, dead zone formation and short-circuiting. Comparative simulations using a model based on the RANS equations were also performed. This is considered particularly important in light of the relatively high computational costs of LES compared with RANS models. Two design variations with different chamber widths are examined. The dimensions and the flow conditions are identical to those of the experimental study performed of scaled model contactors by Kim et al. ("Investigating multi-chamber ozone contactor using 3D laser induced fluorescence," J. Am. Water Works Assoc., in review, 2009).

\section{Numerical Approach}

In this study, simulations were performed applying two different numerical modeling approaches i.e. LES and RANS. The LES is the main focus of this paper and therefore are described in detail. The results from the RANS model are used only for comparison purposes; details of this model are presented in Kim et al. (2009). The LES code (Hydro3D-GT) used in this study is an in-house code that is based on the finite-volume method on a Cartesian grid with collocated variable arrangement. The Hydro3D-GT code solves the following filtered Navier-Stokes equations for incompressible fluid flow (Pope 2005):

$$
\begin{gathered}
\frac{\partial u_{i}}{\partial x_{i}}=0 \\
\frac{\partial u_{i}}{\partial t}+\frac{\partial u_{i} u_{j}}{\partial x_{j}}=-\frac{\partial p}{\partial x_{j}}+\frac{\partial\left(2 \nu S_{i j}\right)}{\partial x_{j}}-\frac{\partial \tau_{i j}}{\partial x_{j}} \\
S_{i j}=\frac{1}{2}\left(\frac{\partial u_{i}}{\partial x_{j}}+\frac{\partial u_{j}}{\partial x_{i}}\right) \\
\tau_{i j}^{a}=\tau_{i j}-\frac{2}{3} \delta_{i j} k_{r}\left(=-2 v_{t} S_{i j}\right) \\
v_{t}=\left(C_{s} \Delta\right)^{2}|S| \\
\Delta=(\Delta x \Delta y \Delta z)^{1 / 3} \\
|S|=\left(2 S_{i j} S_{i j}\right)^{1 / 2}
\end{gathered}
$$

where $u_{i}$ and $u_{j}(i$ or $j=1,2$ or 3$)=$ resolved velocity vectors (i.e. $u_{1}=u, u_{2}=v$, and $u_{3}=w$ denoting the velocity components in $x, y$ and $z$ axis directions, respectively) and $p=$ resolved pressure divided by the density. These quantities are filtered in space. Similarly, $x_{i}$ and $x_{j}$ represent the spatial location vectors in $x, y$, and $z$ axis direction, respectively. $v$ is the kinematic viscosity and $S_{i j}$ is the filtered strain-rate tensor. The $\tau_{i j}$ term results from the unresolved subgrid-scale fluctuations and needs to be modeled by a subgrid-scale (SGS) model. The convection and diffusion terms in the Navier-Stokes equations are approximated by central differences ensuring second-order accuracy in space. An explicit 3 -step Runge-Kutta scheme is used to discretize the equations in time providing third-order accuracy in time. The Smagorinsky SGS model (Smagorinsky 1963) is employed to approximate the anisotropic part via $\tau_{i j}^{a}$ (" $a$ " indicates "anisotropic") where $\delta_{i j}$ is Kronecker delta and $k_{r}$ is the residual kinetic energy. Then, an anisotropic filter, $\Delta$, is used with characteristic filter widths, $\Delta x$, $\Delta y$, and $\Delta z . v_{t}$ is the subgrid-scale eddy viscosity where the Smagorinsky constant, $C_{s}$, is fixed at $0.1 .|S|$ is the characteristic filtered rate of strain.

The model SSIIM (Olsen 2005) was employed to perform the RANS simulations. This model solves the RANS equations with the finite-volume approach on a structured nonorthogonal grid. The SIMPLE method couples the pressure to the velocity field and the standard $k-\varepsilon$ turbulence closure approximates the Reynolds Stresses appearing in the RANS formulation of the NavierStokes equations. A second-order upwind scheme is employed to model the convective terms in the Navier-Stokes equations, whereas diffusive terms are approximated with a centraldifferencing scheme. Details of the model are available in Olsen (2005). 


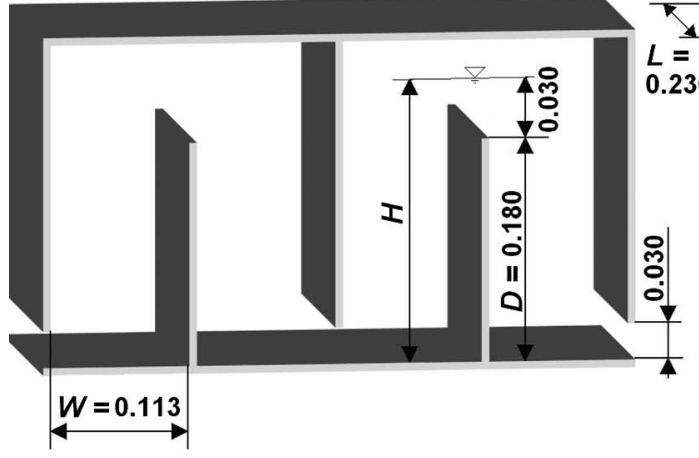

(a)

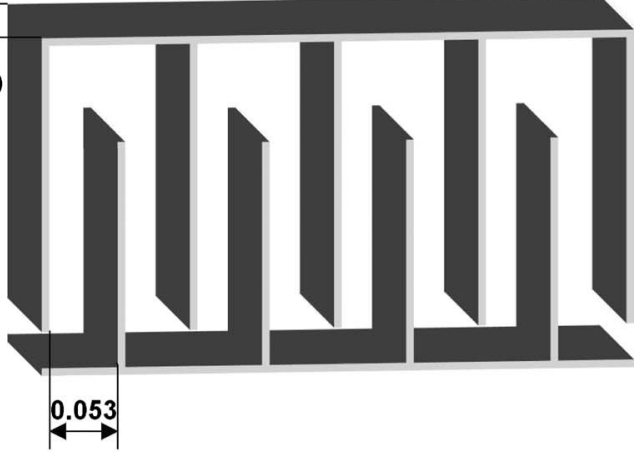

(b)

Fig. 1. Layout of the two model ozone contactors investigated in this study with (a) normal chamber width (NW); (b) half chamber width (HW)

LES and RANS simulations were performed for the laboratory-scale multichamber ozone reactor the flow characteristics of which were extensively evaluated using a 3DLIF technique. In order to reduce the computational cost, the 12 chamber model reactor used by Kim et al. ("Investigating multi-chamber ozone contactor using 3D laser induced fluorescence," J. Am. Water Works Assoc., in review, 2009) was simplified to a four chamber reactor while maintaining the same chamber dimensions. This simplified model is referred to as normal width (NW) model in this study, whereas another reactor with half the channel width, also examined in this study, is referred to as half-width (HW) model. A schematic view of the flow domain including detailed dimensions is provided in Fig. 1.

The computational grid employed in both LES and RANS simulations together with the used boundary conditions for the NW model is presented in Fig. 2. Three preliminary LES on successively finer grids were performed and the results obtained with the finest grid consisting of 1,396,050 total grid points (195 $\times 88 \times 82$ in $x-y-z$ direction) are presented herein. The same grid was used for the RANS model. Note that as the RANS simulations are performed using successively finer grids, the results should become independent of the grid spacing. Such a grid independency is used as a quality indicator for RANS simulations. LES also depends on the employed grid. However, in contrast to RANS simulation, the grid in LES acts as a filter which separates large-scale eddies from small-scale eddies. Hence, in the LES, the grid is required to be fine enough so that the simulation (1) prop-

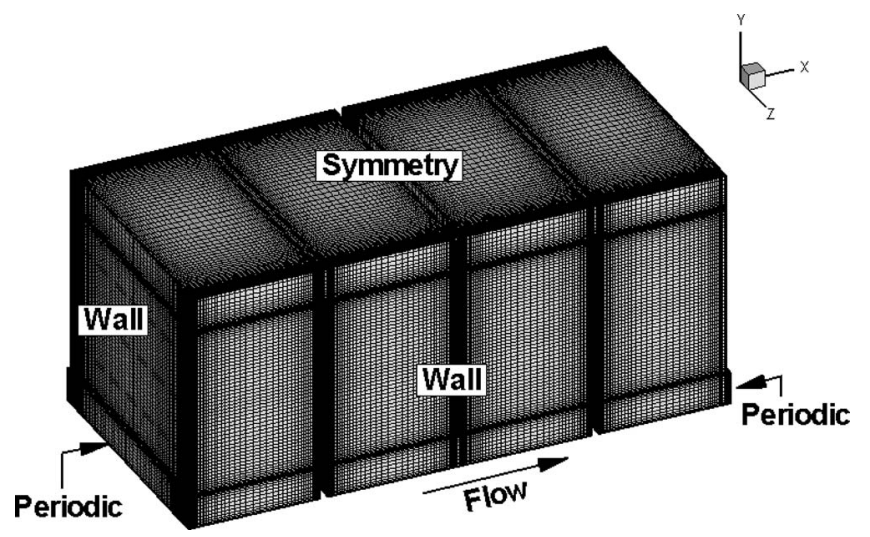

Fig. 2. Grid and used boundary conditions of the numerical models (here: NW reactor) erly captures the largest eddies in the production range of the energy spectra and (2) cascades these eddies within the inertial subrange (see below) in a physically realistic way. It was found that all three grids captured the production range but the finest grid was chosen as it provided the widest range of captured turbulent scales.

The grid is stretched toward the bottom and the baffle walls where velocity gradients are expected to be relatively steep. A no-slip boundary condition (i.e. zero velocity at the wall) is used at all wall boundaries. The grid in the vicinity of the water surface is somewhat coarser than near the wall boundaries, since shear forces are negligible at fluid-air interfaces. Accordingly, a symmetry boundary condition is applied at this free surface. Periodic boundary conditions (i.e. velocities (not scalars) at the exit are copied to the inlet after each time step) are used in the streamwise direction to simulate a geometrically periodic and infinitely long domain. This boundary condition prevents unwanted nonphysical disturbance of the flow which is expected to occur in LES when artificial inflow and outflow boundary conditions are applied. The Reynolds number based on the hydraulic diameter $\left(h_{R}\right.$ $=\sqrt{(4)\left(A_{c}\right) /(\pi)}$, where $A_{c}=$ cross sectional area at the inlet sluice gate) is 2,740 for both models corresponding to one of the experiments performed by Kim et al. ("Investigating multi-chamber ozone contactor using 3D laser induced fluorescence," J. Am. Water Works Assoc., in review, 2009). The LES code uses an adjustable time step that satisfies the Courant-Friedrichs-Lewy stability criterion in every computational cell in order to maintain stability of the explicit time discretization scheme. The time step in the simulations presented here was of the order of 5/1,000 s.

Transport of a conservative tracer was simulated in the LES by solving the following three-dimensional advection-diffusion equation for the filtered tracer concentration, $C$ :

$$
\frac{\partial C}{\partial t}+u_{j} \frac{\partial C}{\partial x_{j}}=D_{t} \frac{\partial^{2} C}{\partial x_{j}^{2}}
$$

where $D_{t}=$ turbulent diffusivity, calculated as the ratio of subgridscale eddy viscosity to the turbulent Schmidt number. The turbulent Schmidt number in the LES was set to 1,000 to maintain the same ratio of molecular viscosity of water to the molecular viscosity of the tracer (Rhodamine 6G) used in the experiments by Kim et al. ("Investigating multi-chamber ozone contactor using 3D laser induced fluorescence," J. Am. Water Works Assoc., in review, 2009). This is justified by the fact that the LES is able to resolve two decades of the energy in the inertial subrange and small-scale turbulence up to frequencies of $100 \mathrm{~Hz}$. The tracer 
was initially introduced at constant concentration at the inlet of the reactor for 500 time steps (i.e. for the duration of approximately $2.5 \mathrm{~s}$ ) to represent a pulse injection. The residence time distribution (RTD) was normalized by the total introduced tracer concentration such that the area under the curve was equal to unity. The time scale was normalized based on the theoretical residence time $(\tau)$ per chamber, which was 27.3 and $12.8 \mathrm{~s}$ for the NW and HW reactors, respectively. The RANS model also solved the convection diffusion Eq. (8) for the transport of a passive scalar. In the slightly modified RANS version of Eq. (8), $u_{j}$ are the Reynolds-averaged velocities and $D_{t}$ is the turbulent diffusivity calculated as the ratio of eddy viscosity (obtained from the $k-\varepsilon$ model) to the turbulent Schmidt number $\left(S_{c}\right)$. In the simulations presented here $S_{c}=0.7$ as suggested in Launder (1978). This is considered a standard value and is also used in commercial codes as a default value (see Fluent user's guide 2003). The computational costs are worth mentioning. In order to obtain a fully developed flow field (i.e. fully developed turbulence in LES and a converged time-averaged flow field in RANS) the RANS simulation was about 5 times faster than the LES on the same grid (2000 iterations in the RANS simulation versus 10,000 time steps in the LES). Once the flow field had been established the tracer simulation of the RANS simulation was about 14 times faster than the one in the LES due to the fact that only one equation needs to be solved $(C)$ while in LES equations for $u, v, w, p$, and $(C)$ need to solved in every time step. Practical RANS computations are expected to have an even greater speed advantage over LES as the RANS grid may not have to be as fine as used in here.

\section{Results and Discussion}

\section{Accuracy of LES Simulation}

Even though the LES approach has proven to be able to accurately predict the flow for a number of different applications, there is still a need for reassessing the accuracy of the LES approach and the numerical methods involved especially in complex flows (Rodi et al. 1997). In this study, commonly used indicators, such as the distribution of dimensionless grid spacing along the walls and the energy spectra of the flow, are used in order to evaluate the quality of the LES. The quality of the near wall grid resolution is assessed based on the distribution of the dimensionless grid spacing, $y^{+}$, defined as follows (Versteeg and Malalasekera 2007):

$$
\begin{gathered}
y^{+}=\frac{d}{v} \sqrt{\frac{\tau_{w}}{\rho}} \\
\tau_{w}=\mu \frac{\bar{U}}{d}
\end{gathered}
$$

where $\tau_{w}=$ wall shear stress; $d=$ distance of the first grid point off the wall; and $\bar{U}=$ time-averaged velocity at the corresponding grid node. $\rho$ and $\mu$ are the density and the dynamic viscosity of fluid, respectively. The no-slip wall boundary condition is the mathematically correct formulation only if the first grid point off the wall is placed within the viscous sublayer (i.e., $y^{+} \leq 11$ ), in which the effects of turbulence are negligible compared to viscous effects. If the first grid point is placed within the viscous sublayer, the highest possible accuracy is achieved. Fig. 3 shows $y^{+}$values near the chamber bottom and the left side wall of a baffle. The values of the first grid point in wall units $y^{+}$are consistently

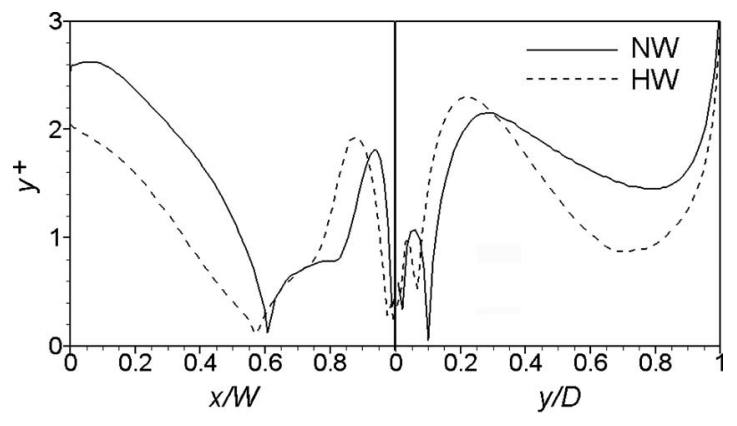

(a)

(b)

Fig. 3. Distribution of $y^{+}$for both models: (a) along the chamber bottom; (b) along the vertical baffle wall

below 3, hence considerably smaller than 11.0 , justifying the noslip wall boundary condition and guaranteeing the accuracy of the LES results.

Power density spectra (PDS) were also examined to evaluate whether the inertial subrange of the turbulent energy transfer was adequately resolved in the present LES. Fig. 4 shows the PDS of the $x$ and $z$ velocity components ( $u$ and $w$, respectively) at selected locations, where the flow is accelerating and the turbulence energy is relatively large. The energy prevailing in large eddies with lower frequency is successively cascaded to smaller eddies with higher frequencies. The simulated decay follows the Kolmogorov slope of $-5 / 3$, when the PDS is plotted versus frequency both in logarithmic scales, confirming that the energy transfer from large scales to small scales is physically realistic. A sudden drop of the spectra represents the virtue of the SGS model dissipating the remaining energy. Noteworthy are peaks in the spectrum of the $u$-velocity signal in the NW reactor at around 200 $\mathrm{Hz}$. These are small-scale numerical oscillations that are caused by the higher-order central-differencing scheme used for the convective terms. These oscillations do not affect the simulation results since they contain very little energy and are rather considered numerical artifacts. Using upwind-based schemes for the convective terms may remove these oscillations. However, they would also introduce numerical dissipation and would lead to considerable less accurate results at the same time.

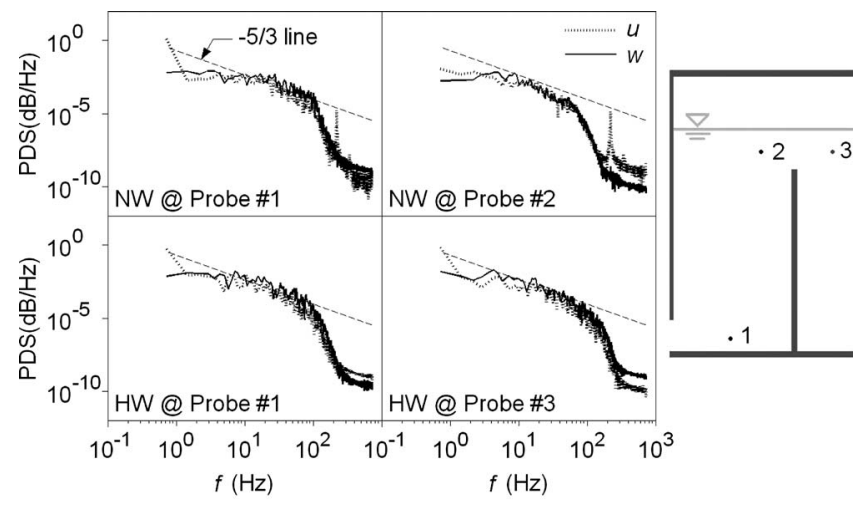

(a)

(b)

Fig. 4. PDS of the LES of " $u$ " and " $w$ " velocities: (a) PDS at three selected points; (b) location of the three points where time signals were sampled 


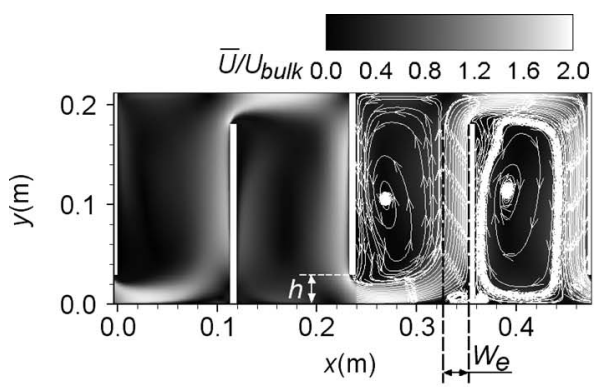

(a)

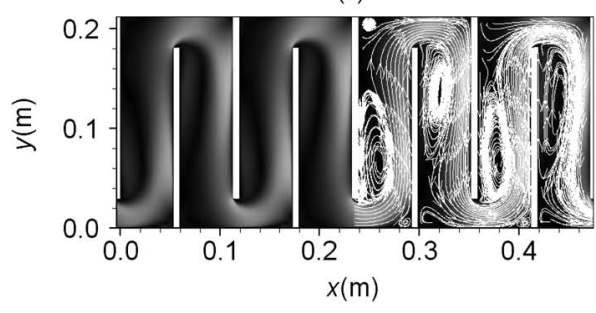

(b)

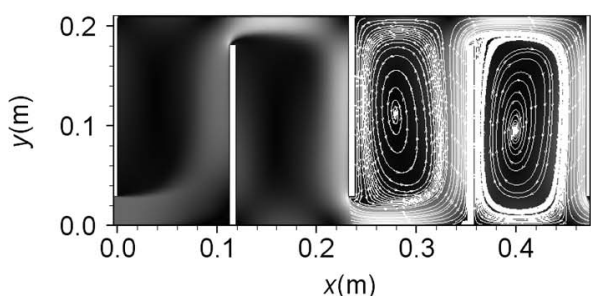

(c)

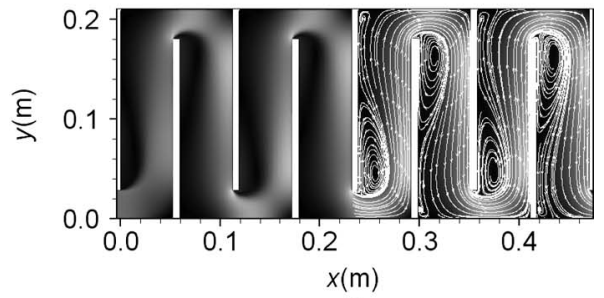

(d)

Fig. 5. Distribution of the time-averaged absolute velocity and streamlines in the center-plane $(z / L=0.5)$ of the camber: (a) NW model (LES); (b) HW model (LES); (c) NW model (RANS); and (d) HW model (RANS)

\section{Flow Characteristics}

Fig. 5 presents the distribution of the time-averaged normalized absolute velocity $\left(\bar{U} / U_{\text {bulk }}\right)$ along with two-dimensional streamlines that are depicted in the right half portion of each figure. Areas of higher speed (white contours) are found along the outer regions of the meandering flow path (i.e. near the right side of each chamber), clearly suggesting the occurrence of shortcircuiting through each chamber. As the flow exits each chamber near the bottom or near the free surface, it is accelerated to twice the average bulk velocity $\left(U_{\text {bulk }}\right)$ due to the presence of the sharp crest of the baffles. The streamlines indicate that a large recirculation zone occupies approximately two thirds of the chamber width in the chambers of the NW reactor [Fig. 5(a)]. Noteworthy is the fact that the effective flow width $\left(W_{e}\right)$ is approximately equal to the gate height $(h)$ in both cases. A small counter-rotating secondary recirculation zone occurs at the corners of the bottom and the baffle walls on either side of the baffle. Such a secondary recirculation zone is not observed near the water surface. A third cell is observed at the downstream side and near the free end of

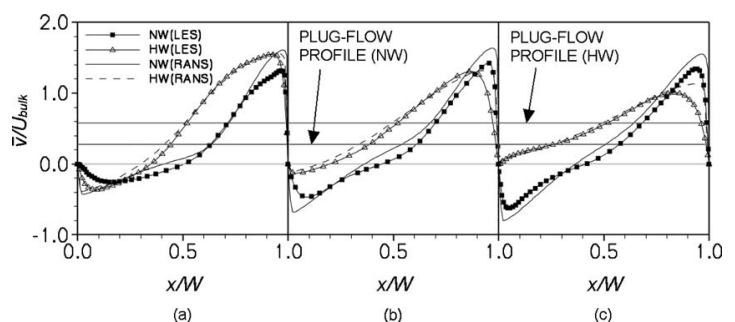

Fig. 6. Time and spanwise-averaged velocity profiles across the chamber width $(W)$ at different depths: (a) $y / H=0.27$; (b) $y / H=0.5$; and (c) $y / H=0.72$

the baffle and its streamlines resemble those of the flow in a lid driven cavity (Ghia et al. 1982). These counter-rotating inner cells are shear-driven by the larger clockwise rotating outer cell.

Although the HW reactor exhibits similar flow features such as similar effective flow width, some differences are noteworthy. In particular, the size of the primary recirculation zone is considerably smaller compared to the NW model. In addition, the primary recirculation zone of the HW model does not occupy the entire chamber height $(H)$, in contrast to the NW model, but extends to only about two thirds of the height of the chamber. Flow acceleration is also observed as the flow passes underneath or over the baffles. The secondary recirculation zone in the bottom/baffle corner is similar to the one found in the NW model, while another secondary cell appears near the free surface. A counterclockwise rotating cell near the free end of the baffles is absent in the HW model suggesting that the shear that is induced by the main cell of the NW model is stronger than that of the HW model.

Figs. 5(c and d) present the Reynolds-averaged velocity contours as predicted by the RANS model for the NW model and the HW model, respectively. The distributions are very similar to the time-averaged velocities predicted by the LES, indicating that the turbulence closure model (i.e. the $k-\varepsilon$ model) is able to capture the effect of large-scale turbulence on the mean flow reasonably well.

Profiles of the LES predicted time- and spanwise-averaged and normalized flow-through velocity $\left(\bar{v} / U_{\text {bulk }}\right.$, i.e. the velocity in the $y$-direction) at three different vertical locations in the chamber for both the NW and the HW reactors are presented in Fig. 6. Also included are the velocity profiles from the RANS simulations. The differences in time-averaged (from the LES) and Reynoldsaveraged (from the RANS simulations) velocity profiles are fairly small, particularly in the HW model, although the RANS model seems to overestimate the near wall gradients compared to the LES with the maximum deviations in the order of $10-15 \%$. The calculated velocity profiles clearly deviate from those of plug flow (i.e. uniform velocity profile), indicating the presence of severe short-circuiting (i.e. flow with locally high velocity near one wall) and recirculation (i.e. negative velocities at near the opposite wall). Close to the chamber bottom [Fig. 6(a), $y / H=0.27$, the absolute values of the velocity peaks are smaller in the NW reactor compared to the HW reactor. The area over which recirculation occurs is considerably larger in the NW reactor. At half water depth $(y / H=0.5)$, there is yet faster moving fluid near the right wall in both models, but the recirculation has almost disappeared in the HW reactor. At $y / H=0.72$, the NW model still shows the same trend with a large velocity difference between the left and right wall flows, while in the HW reactor the reverse flow has disappeared and the velocity profile is closer to that of the plug flow. 


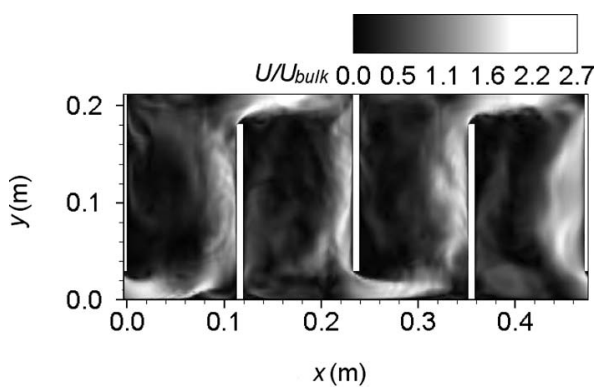

(a)

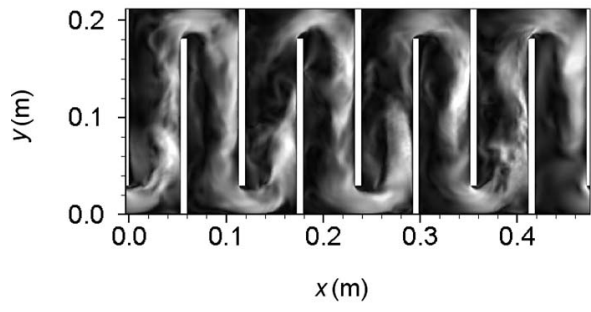

(b)

Fig. 7. Distribution of instantaneous velocities in the center-plane of the chamber $(z / L=0.5)$ : (a) NW model; (b) HW model

Fig. 7 presents the distribution of the LES calculated instantaneous normalized speed $\left(U / U_{\text {bulk }}\right)$ profiles in both reactors. Although the instantaneous velocity distribution overall resembles the time-averaged velocity distribution presented in Fig. 5, the prevalence of turbulence and unsteadiness is apparent. In particular, smaller vortices are observed in both reactors, especially along the shear layers that form between the main flow path and the recirculation region. In the HW reactor, these vortices lead to a partial disruption of the main flow path. The instantaneous velocity in the main flow path reaches about 2.2-2.7 times the bulk velocity, again indicating severe short-circuiting in both reactors.

A more quantitative view of instantaneous normalized flowthrough profiles predicted using the LES at three different instants in time and at three different vertical locations in one chamber of the NW (upper figures) and HW (lower figures) models are provided in Fig. 8. The time-averaged profiles are also plotted in Fig. 8. At any given instant in time, the instantaneous velocity deviates clearly from the mean value and the instantaneous velocity maxima are either above or below the mean values demonstrating

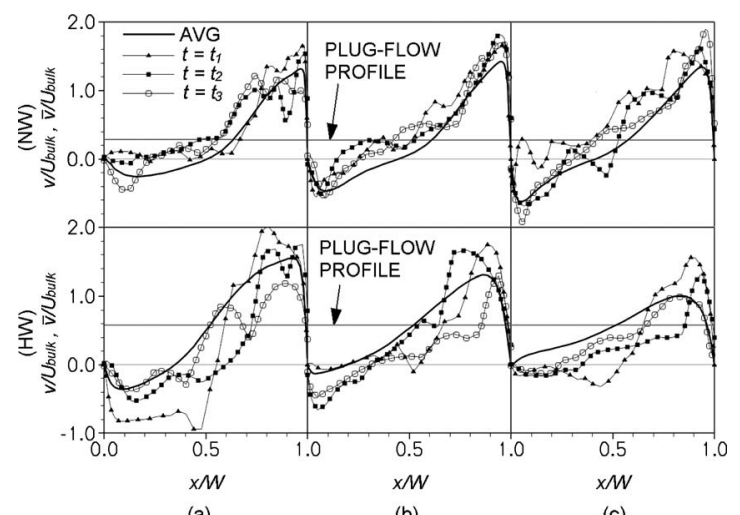

(a)

(b)

(c)

Fig. 8. Instantaneous velocity profiles across the chamber width $(W)$ at different depths: (a) $y / H=0.27$; (b) $y / H=0.5$; and (c) $y / H=0.72$

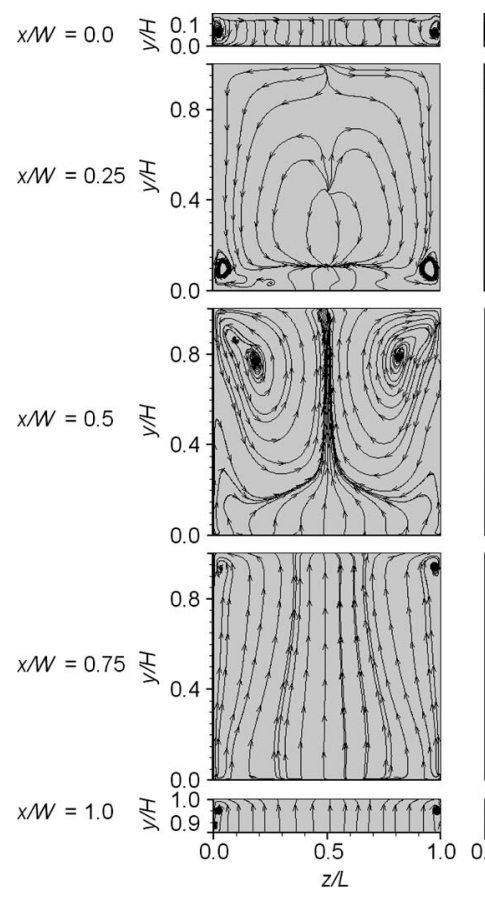

(a)
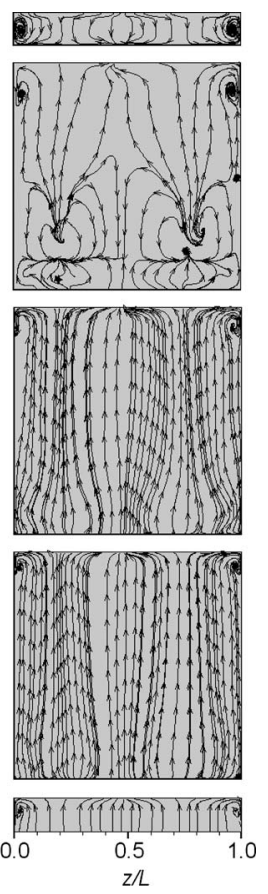

(b)
Fig. 9. Time-averaged streamlines in selected spanwise planes: (a) NW model; (b) HW model

turbulence and intermittency of the flow. The maxima of instantaneous velocity are about 5 times higher than the plug flow value in the NW chamber, but the velocity maxima are "only" about 3 times higher in the HW reactor. At $y / H=0.72$, this ratio is still approximately 5 in the NW model while it has decreased to a factor of 2.2 in the HW model, which is mainly due to the absence of recirculation at this depth. More details of statistics of velocity fluctuations (i.e., rms values of the velocity) are provided in Kim et al. (2009).

Streamlines of the secondary flow in $y-z$ planes at five different streamwise locations within the first chamber are shown in Fig. 9. These suggest that the flow field is highly threedimensional. At $x / W=0.0$ (i.e. the plane of the reactor entrance), two small countercirculating vortices near the side walls are observed in both reactors. At $x / W=0.25$, these vortices are still present in the NW model and a general downward movement (i.e. as a result of the main recirculation zone) is observed. There is a nodal point at $z / L=0.5$ and $y / H=0.42$ at which streamlines diverge. This nodal point corresponds to the center of the main streamwise recirculation zone. Along the upper side of the baffle opening (i.e. $y / H \approx 0.1$ ), the streamlines from the recirculation zone and those from incoming fluid converge. In contrast, the small vortices near the entrance region are dissipated at $x / W$ $=0.25$ and a new recirculation zone forms near the free surface in the HW reactor. Converging streamlines, similar to the NW reactor, are found along the baffle opening height and a pair of nodal points corresponding to the center of the streamwise recirculation zone is also found relatively close to the chamber bottom $(y / H$ $\approx 0.2$ ). Above approximately half the chamber depth (i.e. $y / H$ $>0.5$ ), the flow exhibits an upward motion. At half the width (i.e. $x / W=0.5$ ), a pair of large, symmetric, and counter-rotating vortices are observed in the NW reactor again suggesting threedimensionality and fluid entrainment. Near the bottom of the chamber, the fluid exhibits an upward movement over the entire 


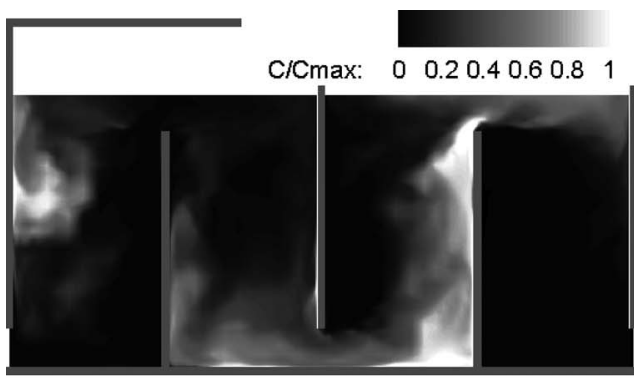

(a)

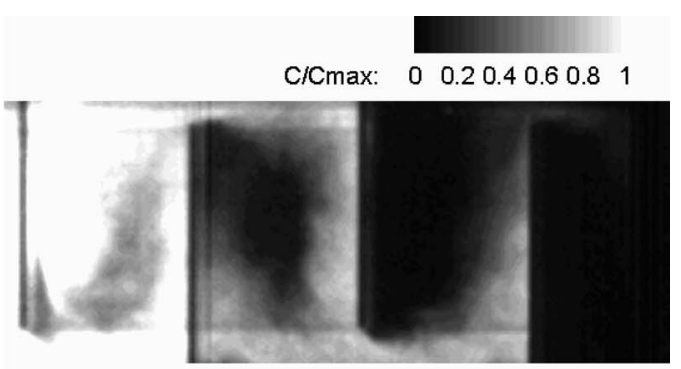

(b)

Fig. 10. Computed (left) and measured (right) tracer distribution in the chamber at an instant in time

width. The secondary streamlines of the HW model suggest that the flow is almost two-dimensional at $x / W=0.5$. At $x / W=0.75$ and 1.0 for both reactors, upward motion is found over the entire chamber width. The small vortices near the free surface are almost dissipated at $x / W=1.0$.

\section{Tracer Transport}

Fig. 10 presents snapshots of the distribution of instantaneous normalized tracer concentration as predicted by the LES (left) and as observed in the laboratory (right). The experimental 3DLIF image was adopted from Kim (2007). It has to be noted that, although the flow conditions are identical, the amount of tracer introduced into the reactor is less in the LES, explaining the discrepancy between experiment and simulation (especially in the first chamber). Nevertheless, there is a fairly good qualitative agreement between the observed and the predicted concentration distributions in the second to fourth chambers. Higher concentration (brighter contour) is found near the baffles or near the free surface, whereas lower concentration (darker contour) prevails in the center of the recirculation zones. The concentration distributions support the occurrence of the aforementioned flow features i.e. short-circuiting and occurrence of a large recirculation region in each chamber.

Fig. 11 shows the instantaneous distribution of the isoconcentration surface (i.e. the surface with tracer concentration higher than $1 \%$ of input concentration) as a function of time. These images again confirm the nonideal flow in the reactor. The shortcircuiting is observed as a distinctive plume, for example, in the second chamber of the NW reactor (upper series) at $t=10.1 \mathrm{~s}$. Internal recirculation and the accompanying dead zone are apparent in the images taken at $90.5 \mathrm{~s}$ (the first chamber) and $114.2 \mathrm{~s}$ (the second chamber). As a result of nonideal flow, the tracer is widely distributed across the NW reactor: some parts of the injected tracer have already escaped the reactor while other parts are still present in the inlet region (e.g., images at $t=23.5$ and 43.7 $\mathrm{s})$. Consistent with the previous hydrodynamic observations, the short-circuiting is less severe in the HW model. Consequently, the tracer is less widely distributed across the HW reactor compared with the NW reactor. For example, at $t=10.1 \mathrm{~s}$, the tip of the tracer plume in the NW reactor already reached the half of the volume of the reactor, whereas the front of the tracer plume in the HW model has just passed through in the second chamber. At $t$ $=23.5 \mathrm{~s}$, the tracer in the NW model is about to exit the reactor, while in the HW reactor the tracer is only halfway through. At $t$ $=114.2 \mathrm{~s}$, while tracer still occupies 3 out of 4 chambers in the NW model, most of the tracer has already left the HW reactor.

\section{Residence Time Distribution Analysis}

Fig. 12(a) presents the RTDs experimentally determined by Kim et al. ("Investigating multi-chamber ozone contactor using 3D laser induced fluorescence," J. Am. Water Works Assoc., in review, 2009) and those simulated by the LES and RANS models for both reactors. Note that the reactor used in the experiment consists of 12 chambers with chamber width equal to that of the NW reactor. Since the periodic boundary condition used in the present LES simulation does not accurately represent the inlet flow condition of the laboratory experiments, the RTDs for the first chamber do not agree well. However, magnitude and shape of the LES computed RTD curves match the observations very well from the second chamber onward where the effect of the boundary condition is almost negligible. In particular, the presence of a sharp short-circuiting peak and a secondary peak that result from internal recirculation (i.e. the tracer exiting the cham-

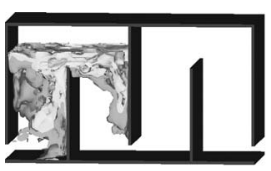

$t=10.1 \mathrm{~s}$

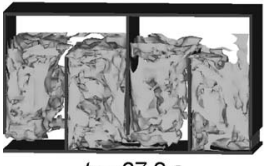

$t=67.2 \mathrm{~s}$

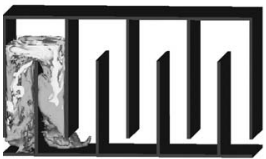

$t=10.1 \mathrm{~s}$

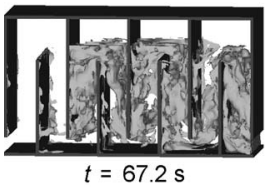

$t=67.2 \mathrm{~s}$

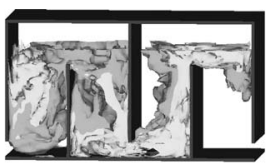

$t=23.5 \mathrm{~s}$

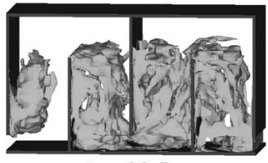

$t=90.5 \mathrm{~s}$

(a)

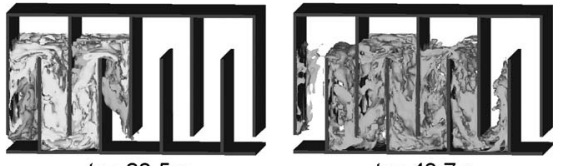

$t=23.5 \mathrm{~s}$

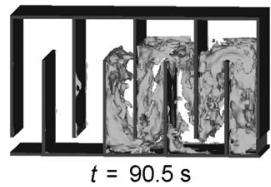

(b)

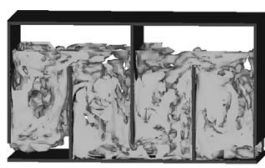

$t=43.7 \mathrm{~s}$

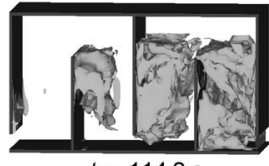

$t=114.2 \mathrm{~s}$

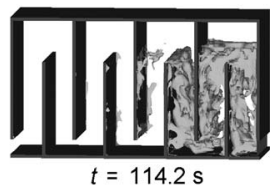

$t=114.2 \mathrm{~s}$
Fig. 11. Isosurface of tracer concentration at six selected instants in time: (a) NW model; (b) HW model 


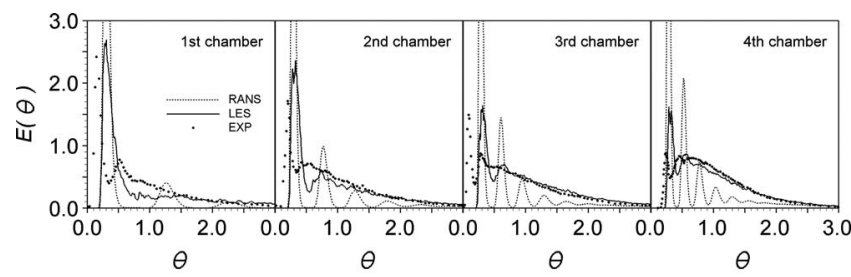

(a)

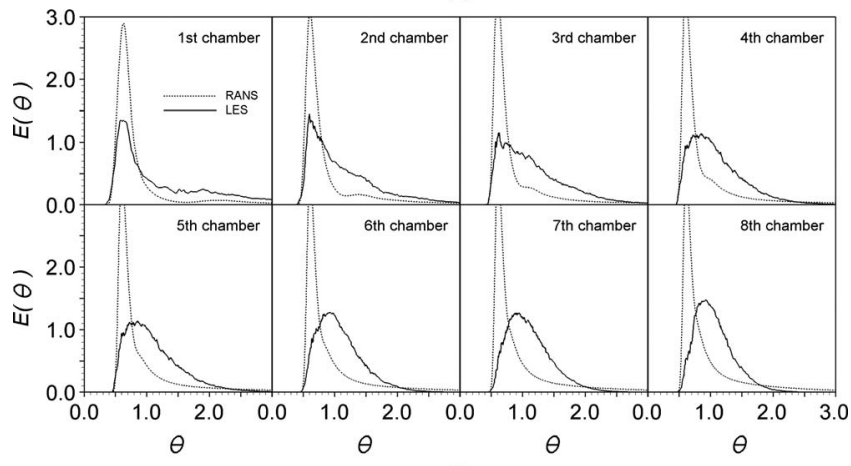

(b)

Fig. 12. Tracer RTD curves at the exit sluice gate of each chamber: (a) NW model; (b) HW model

ber after single recirculation) are noticeable in both experimental RTDs and LES RTDs (for the NW reactor).

The RTD curves obtained from the RANS simulations show much more severe short circuiting and also exhibit pronounced secondary recirculation peaks. The unsteadiness and intermittency of the flow are not fully expressed in the RTDs obtained from the RANS simulations which deviate largely from those measured or computed by the LES. Since the RANS model predicts the same Reynolds-averaged flow for every chamber and for every time step of the tracer transport computation, the secondary recirculation peaks appear very regularly in time. The peaks are obviously expressing the entrapment of tracer in the recirculation zones and subsequent release of tracer from the recirculation zones.

The RTD curves for the HW reactor obtained by both LES and RANS models show also noticeable differences [Fig. 12(b)]. The LES calculated RTD from the first chamber is characterized by a single short-circuiting peak that occurs slightly later than that in the NW reactor. No secondary peak is detectable as internal recirculation is less severe. The RTD curves obtained at later chambers gradually become a symmetrical Gaussian-shaped curve centered at around $\theta=1.0$. Overall, the RTD curves become closer to that of a plug flow reactor (PFR) as the number of chambers increases, diluting the effect of nonideal mixing condition in each chamber (i.e. gradual disappearance of short-circuiting peak). The LES simulation clearly suggests that the reactor with narrower chamber width gives a RTD that is much closer to that of a PFR than the reactor with wider chamber width. The RTD as calculated by the RANS model exhibits a much higher peak that is maintained through all eight chambers. These early peaks suggest that most of the introduced tracer is transported along the shortcircuiting flow path with little tracer being trapped in recirculation zones. In both cases (the NW and HW reactors), it might be possible to improve the RANS model predictions by calibrating the turbulent diffusivity of the tracer. This is considered beyond the scope of this study.

Cumulative RTD $(F)$ curves are obtained by integrating the RTD curves in Fig. 12, and the $t_{10} / \tau$ values are evaluated (Fig. 13). Note that $t_{10} / \tau$ represents the time required for $10 \%$ of the

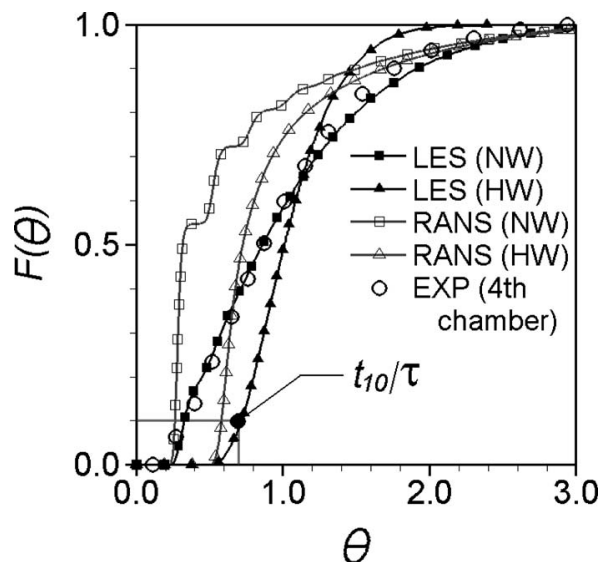

Fig. 13. Cumulative tracer RTD curves at the exit gate of each contactor

tracer (injected as a pulse) to exit the reactor. This is commonly used as a characteristic time to evaluate the disinfection credit according to the current regulations (U.S. EPA 1999). Two critical conclusions can be drawn from this analysis. First, the $t_{10} / \tau$ value of the NW reactor predicted by the LES is 0.3 , which is much smaller than the $t_{10} / \tau=0.7$ value of the HW reactor. According to the disinfection reactor baffle classification by the U.S. EPA (1999), the NW reactor would be classified as a poor baffling condition reactor in which no baffle is typically installed. This result suggests that even though the reactor is designed as a multichamber, the occurrence of nonideal mixing behavior could have a detrimental effect on the overall hydrodynamics of the reactor when the baffle configurations are not properly designed. For example, Crozes et al. (1998) carried out an experimental and numerical study on a multichamber chlorine contactor and showed that the $t_{10} / \tau$ values increase with an increase in the number of baffles over a defined contactor length. A recent study by Kim et al. (2007) suggested that a full-scale ozone contactor with 10 chambers was best represented with a cascade of only 7 continuous stirred tank reactors. The HW reactor, according to the same classification, would be considered as the superior baffling reactor. With respect to regulatory compliance (U.S. EPA 1999), this reactor is preferred for a greater disinfection credit. This flow condition also helps minimizing the process overdesign and reduces the formation of disinfection by-products such as bromate (Roustan et al. 1993; Do-Quang et al. 2000; Kim et al. 2007). Second, the $t_{10} / \tau$ value obtained from the LES matched well with that experimentally measured. In contrast, the RANS model predicts that $t_{10} / \tau=0.22$ for the NW reactor and $t_{10} / \tau=0.58$ for the HW reactor, which are much smaller than the values obtained with the LES. Since the regulatory disinfection credit is based on $t_{10} / \tau$, reactor design based on the RANS simulation would result in a significant (e.g., over $25 \%$ in NW reactor) underestimation of $t_{10} / \tau$ and consequently overdesign of the process (in terms of increased ozone dose and/or increase reactor volume).

\section{Conclusions}

The LES results suggest the occurrence of deficient flow conditions and nonideal solute transport behavior in the two multichamber ozone contactors examined in this study. The flow through these reactors is characterized by the presence of extensive short-circuiting and large internal recirculation that cause the 
occurrence of a dead zone in each chamber. These observations are consistent with the experimental findings by Kim et al. ("Investigating multi-chamber ozone contactor using 3D laser induced fluorescence," J. Am. Water Works Assoc., in review, 2009), who carried out tracer studies in a scale reactor that had the same dimensions and flow rate as used in this study. The LES results suggest that the flow is highly three-dimensional with a pair of symmetric counter-rotating secondary vortices and nodal points in the center of the recirculation zones. The RTDs evaluated at several locations in the reactors confirm that the chamber width of an ozone contactor is an important design parameter.

Considering that multichamber design is intended to provide plug flow conditions, above findings conclude that careful design is necessary to avoid the occurrence of nonideal hydrodynamics and significant deviation of the flow from the original design intent. The LES results show that these hydrodynamic deficiencies could be partially prevented by decreasing the chamber width. The present LES was performed for only two representative designs and other design options such as the dimension of the baffle inlet height and flow rate need to be studied further. Nevertheless, this study showed that LES can be a useful tool for new plant design as well as retrofitting existing reactors for better process efficiency.

Additional simulations performed with a RANS model demonstrate both similarity and difference between these two modeling approaches. Although Reynolds-averaged velocities from the RANS model match closely with time-averaged velocities obtained from the LES, the RANS model tracer transport predictions differ significantly from both LES prediction and experimental measurement. Underestimation of characteristic residence time, $t_{10} / \tau$, which is used for disinfection credit evaluation, by the RANS model could lead to process overdesign.

\section{Acknowledgments}

The research is partially funded by the U.S. Environmental Protection Agency (Cooperative Agreement Nos. CR-83327701 and 834118). Mention of trade names or commercial products does not constitute endorsement or recommendation for use. The scientific views expressed are solely those of the writers and do not necessarily reflect those of USEPA. We thank the reviewers of the original manuscript for their valuable comments, which helped improving this paper.

\section{Notation}

The following symbols are used in this paper:

$A_{c}=$ cross-sectional area at the inlet sluice gate;

$C=$ instantaneous tracer concentration;

$C_{s}=$ Smagorinsky constant;

$D=$ lower baffle height;

$D_{t}=$ turbulent diffusivity;

$d=$ normal distance from a wall;

$E=$ normalized RTD;

$F=$ cumulative RTD;

$f=$ frequency;

$H=$ water surface level;

$h=$ the height of sluice gate;

$h_{R}=$ hydraulic radius;

$k_{r}=$ residual kinetic energy;

$L=$ thickness of reactor in $z$ direction;

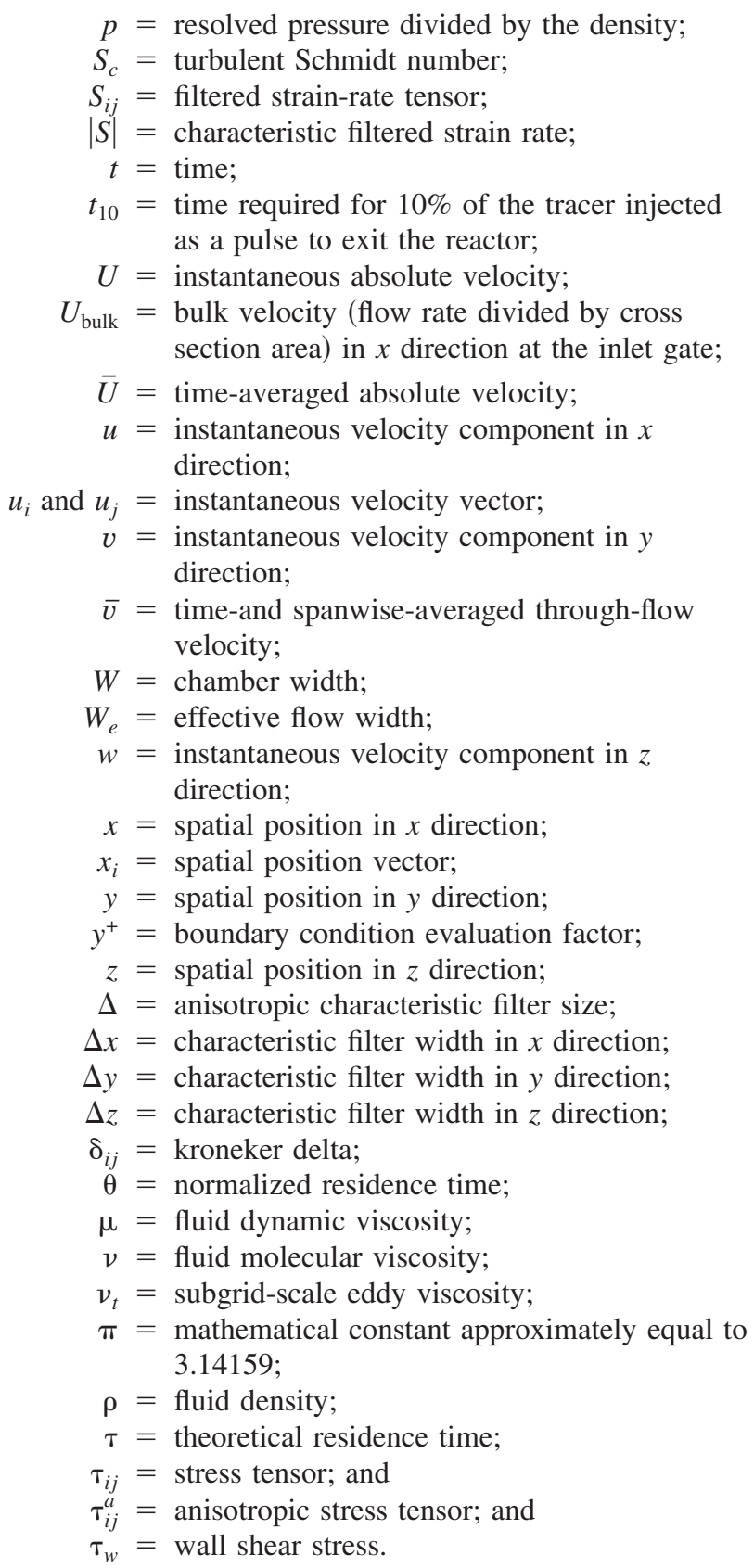

\section{References}

Crozes, G. F., Haqstrom, J. P., Clark M. M., Ducoste, J, and Burns, C. (1998). "Improving clearwell design for CT compliance." AWWA RF Rep. No. 90756, AWWA, Denver.

Do-Quang, Z., Ramirez, C. C., and Roustan, M. (2000). "Influence of geometrical characteristics and operating conditions on the effectiveness of ozone contacting in fine-bubbles conventional diffusion reactors." Ozone: Sci. Eng., 22(4), 369-378.

Fluent user's guide version 6.1. (2003). Fluent, Lebanon, N.H., 〈http:// 202.185.100.7homepagefluenthtml/ugnode314.htm $\rangle$.

Ghia, U., Ghia, K. N., and Shin, C. T. (1982). "High-resolution for incompressible flow using the Navier-Stokes equations and a multi-grid method." J. Comput. Phys., 48, 387-411.

Gualtieri, C. (2006a). "Numerical simulations of flow and tracer transport in a disinfection contact tank." iEMSS 2006, iEMSs, Burlington, Vt.

Gualtieri, C. (2006b). "Numerical simulation of RTD in contact tanks with COMSOL multiphysics." Proc., COMSOL User Conf. 2006, COMSOL AB, Napoli, Italy. 
Gualtieri, C. (2007). "Analysis of the effect of baffles number on a contact tank efficiency with multiphysics 3.3." Proc., COMSOL User Conference 2007, COMSOL AB, Napoli, Italy.

Heathcote, G. R. (1995). "Development of an ozone disinfection contactor using a physical scale model." Ozone: Sci. Eng., 17(1), 15-24.

Henry, D. J., and Freeman, E. M. (1995). "Finite element analysis and T-10 optimization of ozone contactors." Ozone: Sci. Eng., 17(6), 587-606.

Kim, D., Kim, J. H., and Stoesser, T. (2009). "LES of flow in an ozone contactor: Mean and instantaneous turbulent flow characteristics." Proc., IAHR Conf. 2009, Vancouver, Canada.

Kim, D. I. (2007). "Development and application of integrated ozone contactor design and optimization tools." Ph.D. thesis, Georgia Institute of Technology, Atlanta.

Kim, D. I., Tang, G., Hasan, S., Mariñas, B. J., Couillard, L., Shukairy, H. M., and Kim, J. H. (2007). "Simultaneous simulation of pathogen inactivation and bromate formation in a full-scale ozone contactor by computer software." J. Am. Water Works Assoc., 99(8), 77-91.

Kim, J. H., von Gunten, U., and Mariñas, B. J. (2004). "Modeling bromate formation and Cryptosporidium parvum inactivation in synthetic waters." Environ. Sci. Technol., 38(7), 2232-2241.

Launder, B. E. (1978). "Heat and mass transport." Turbulence, Chap. 6, P. Bradshaw, ed., Springer, Berlin.

Murrer, J., Gunstead, J., and Lo, S. (1995). "The development of an ozone contact tank simulation model." Ozone: Sci. Eng., 17(6), 607617.

Olsen, N. R. B. (2005). SSIIM user's manual, Dept. of Hydraulic and Environmental Engineering, The Norwegian Univ. of Science and Technology, 〈http://www.ntnu.no/ nilsol/ssiimwin/manual3.pdf $\rangle$.

Pope, S. B. (2005). Turbulent flows, Cambridge University, Cambridge,
U.K., 219-249, 581-582.

Rodi, W., Ferziger, J. H., Breuer, M., and Pourquie, M. (1997). "Status of large eddy simulation: Results of a workshop.” ASME J. Fluids Eng., 119(2), 248-262.

Rogallo, R. S., and Moin, P. (1984). "Numerical simulation of turbulent flows.” Annu. Rev. Fluid Mech., 16, 99-137.

Roustan, M., Beck, C., Wable, O., Duguet, J. P., and Mallevialle, J. (1993). "Modeling hydraulics of ozone contactors." Ozone: Sci. Eng., 15(3), 213-226.

Shiono, K., and Teixeira, E. C. (2000). "Turbulent characteristics in a baffled contact tank." J. Hydraul. Res., 38(4), 271-416.

Smagorinsky, J. (1963). "General circulation experiments with the primitive equations, part I: The basic experiment." Mon. Weather Rev., 91(3), 99-164.

Tang, G., Adu-Sarkodie, K., Kim, D., Kim, J. H., Teefy, S., Shukairy, H. M., and Mariñas, B. J. (2005). "Modeling Cryptosporidium parvum oocyst inactivation and bromate formation in a full-scale ozone contactor." Environ. Sci. Technol., 39(23), 9343-9350.

U.S. EPA. (1999). "Disinfection profiling and benchmarking guidance manual, Appendix A.” Rep. No. EPA-815-R-99-013, U.S. EPA, Washington, D.C.

Versteeg, H. K., and Malalasekera, W. (2007). An introduction to computational fluid dynamics: The finite volume method, Pearson Education, England, 273-275.

Wang, H., and Falconer, R. A. (1998a). "Simulating disinfection processes in chlorine contact tanks using various turbulence models and high-order accurate difference schemes." Water Res., 32(5), 1529 2543.

Wang, H., and Falconer, R. A. (1998b). "Numerical modeling of flow in chlorine disinfection tanks." J. Hydraul. Eng., 124(9), 918-931. 